\title{
Carbon Micronymphaea: Graphene on Vertically Aligned Carbon Nanotubes
}

\author{
Jong Won Choi, Seul Ki Youn, and Hyung Gyu Park \\ Institute of Energy Technology, ETH Zürich, Sonneggstraße 3, 8092 Zürich, Switzerland \\ Correspondence should be addressed to Hyung Gyu Park; parkh@ethz.ch
}

Received 5 March 2013; Accepted 24 July 2013

Academic Editor: Nadya Mason

Copyright (C) 2013 Jong Won Choi et al. This is an open access article distributed under the Creative Commons Attribution License, which permits unrestricted use, distribution, and reproduction in any medium, provided the original work is properly cited.

This paper describes the morphology of carbon nanomaterials such as carbon nanotube (CNT), graphene, and their hybrid structure under various operating conditions during a one-step synthesis via plasma-enhanced chemical vapor deposition (PECVD). We focus on the synthetic aspects of carbon hybrid material composed of heteroepitaxially grown graphene on top of a vertical array of carbon nanotubes, called carbon micronymphaea. We characterize the structural features of this unique nanocomposite by uses of electron microscopy and micro-Raman spectroscopy. We observe carbon nanofibers, poorly aligned and well-aligned vertical arrays of CNT sequentially as the growth temperature increases, while we always discover the carbon hybrids, called carbon micronymphaea, at specific cooling rate of $15^{\circ} \mathrm{C} / \mathrm{s}$, which is optimal for the carbon precipitation from the Ni nanoparticles in this study. We expect one-pot synthesized graphene-on-nanotube hybrid structure poses great potential for applications that demand ultrahigh surface-to-volume ratios with intact graphitic nature and directional electronic and thermal transports.

\section{Introduction}

Unique properties of carbon nanotubes (CNTs) and graphene have allowed these nanocarbon allotropes to draw great attention in electronic devices, efficient heat and electron transfer media, and nanometric mechanical structures. Both $\mathrm{CNT}$ and graphene have long electron mean free paths $[1,2]$, large carrier mobilities $[3,4]$ at room temperature, excellent mechanical strengths $[5,6]$, and thermal conductivities $[7,8]$ much higher than any other heat conductors. A hybrid system made of CNT and graphene can be a three-dimensional conductive carbon network and thus advantageous for the reduction of the charge transfer resistance through CNTs, the transparency enhancement of CNT electrode, and the remarkable capacity delivery along the basal plane of graphene with a hold of each material's merit. For instance, the early numerical study on the carbon composite between a graphite and CNTs by Paulson et al. [9] has shown that the discrete Fermi surfaces allow electrons to move in a specific direction and make the contact resistance changed sensitively with respect to the lattice angle, thereby improving the conductance along the graphite by CNTs. By understanding the synergistic effect on electrical conductivity among CNTs graphite, graphene, and their hybrids, researchers began to design an electronic device prototype including junction structures between graphene and tangentially dispersed CNTs, and experimentally verified the superiority of the graphene/CNT composite by demonstrating the improved electrochemical cell performance such as solar cell, supercapacitor, and battery by reporting lower sheet resistance, higher specific surface area, higher transmittance, higher reversibility, and a nearly rectangular cyclic voltammetric motion at an exceedingly high scan rate [10-15]. Despite these efforts, the comprehensive understandings of the optimal synthesis conditions and potential applications of the graphene-vertically aligned CNT hybrid are still not well defined because the aforementioned studies used the solution processing or mechanical approaches to make the hybrid composite. Recently, only few studies have been reported on the nanocomposite synthesis from graphene and vertically aligned CNTs and the growth mechanisms based on chemical vapor deposition (CVD). Those researchers have used Co film [16] and Ni film [17] as catalysts for synthesizing both CNTs and graphene layer on top under the thermal CVD method, depending on the initial catalyst thickness and annealing condition. The authors reported that they synthesized the multi- or 
few-layered graphene by feeding a carbon precursor from the beginning during increasing the temperature up to $600^{\circ} \mathrm{C}$ without initial annealing process, and then they subsequently grew the carbon nanotubes after cleaving the catalyst film into nanoparticles above the temperature of $400^{\circ} \mathrm{C}$. It is in general accepted that carbon nanostructure is classified into three regimes, based on the prepared catalyst thickness: singleor double-walled carbon nanotubes on the thin catalyst less than $1 \mathrm{~nm}$ [18-20], precipitated graphene on two orders of magnitude thicker catalysts [21-23], and Carbon nanofibers (CNFs) or multiwalled carbon nanotubes (MWCNTs) on the intermediately sized catalyst [24-26], meaning that the initial catalyst thickness is one of the key factors to decide the shape of carbon allotropes. Here, two questions arise. Are we able to synthesize the three dimensional graphene/vertically aligned CNT composite based on the intermediately thick catalyst? In addition, do the dissolved carbon atoms inside the catalyst particles precipitate to form the graphene layer? To explore the questions, we accordingly made our strategy to use: (1) the intermediately sized $7 \mathrm{~nm}$ thick $\mathrm{Ni}$ film as an initial catalyst, (2) plasma-enhanced CVD to provide more amount of the active carbon species into the catalyst particles, and (3) control of the growth temperature and the cooling rate of the chamber as a one-pot synthesis method. We expect the carbon micronymphaea has enormous potentials such as three-dimensional electrodes, three-dimensional heat dissipation device, and alternatives of metal brush for the sliding electrical contacts.

\section{Materials and Methods}

The unordinary microcarbon Nymphaea structure was synthesized from a bilayer catalyst composed of $7 \mathrm{~nm} \mathrm{Ni}$ and $20 \mathrm{~nm}$ Al layers on a Si substrate via a one-step, plasmaenhanced chemical vapor deposition process in a commercial system (Aixtron). Electron beam evaporation (Univex 450, Leybold) was used to prepare the bilayer catalyst on a $\mathrm{Si}$ substrate.

Prior to the growth, the CVD chamber was cleaned using $\mathrm{O}_{2}$ plasma at $500^{\circ} \mathrm{C}$ for 5 minutes. Then, the catalyst substrates were loaded onto the substrate heater, and the chamber was evacuated below $2 \times 10^{-3}$ mbar initially. The reactor temperature was kept above $200^{\circ} \mathrm{C}$ during loading/unloading to minimize moisture condensation out of surroundings. Under $200 \mathrm{sccm}$ of $\mathrm{NH}_{3}$ and $80 \mathrm{~W}$ of DC plasma at $500 \mathrm{~V}$, the reactor was raised to the growth temperature $\left(550^{\circ} \mathrm{C}\right.$, $650^{\circ} \mathrm{C}$, and $750^{\circ} \mathrm{C}$ ) at a ramp rate of $5^{\circ} \mathrm{C} / \mathrm{s}$ and maintained there for $5 \mathrm{~min}$ for catalyst reduction. The fast ramping is known to defer catalyst sintering unfavorable for catalytic activity and nucleation density [27]. During the $15 \mathrm{~min}$ long growth step, $50 \mathrm{sccm}$ of $\mathrm{C}_{2} \mathrm{H}_{2}$ (high purity > 99.6\%) was added to the $\mathrm{NH}_{3}$ flow under $120 \mathrm{~W}$ of DC discharge power at $500 \mathrm{~V}$. Upon completion of growth, the reactor was cooled down at a desired linear rate under $\mathrm{N}_{2}(10,000 \mathrm{sccm})$ flow and auxiliary heating to fit the cooling rate. The chamber pressure was maintained constant at $12 \mathrm{mbar}$ throughout both annealing and growth processes and 37 mbar during the cooling process, respectively. Since one of the important control parameters of our study is the sample cooling rate, we maintained a linear cooling curve by combined use of the fastest convective $\mathrm{N}_{2}$ cooling and auxiliary PID-controlled heating. In this way, precise linear cooling rate was obtained between $5^{\circ} \mathrm{C} / \mathrm{s}$ and $25^{\circ} \mathrm{C} / \mathrm{s}$ up to the chamber temperature of $200^{\circ} \mathrm{C}$. After the CVD chamber cools down to $200^{\circ} \mathrm{C}$, morphologically various carbon nanostructures were synthesized. Subsequently, the resultant carbon nanostructures were characterized by scanning electron microscopy (SEM, Zeiss Ultra 55), transmission electron microscopy (TEM, Philips CM 12 at $100 \mathrm{keV}$ ), and micro-Raman spectroscopy (WiTec CRM 200 (532 nm excitation)) with a 100x objective lens.

\section{Results and Discussion}

Figure 1 illustrates various morphologies of carbon nanofibers, CNTs, and graphene-nanotube composite, including the growth condition. Since the relatively thick metal layer $(7 \mathrm{~nm} \mathrm{Ni})$ at low temperatures is hard to cleave into a granular film necessary for growing a dense array of CNT $[28,29]$, we obtained only a mixture of poorly aligned, large diameter multiwall CNTs and some carbon nanofibers at $550^{\circ} \mathrm{C}$ (Figures $1(\mathrm{a})-1(\mathrm{c})$ ). The vertical alignment at $650^{\circ} \mathrm{C}$ (Figures 1(d)-1(f)) and the dense array of MWCNT at $750^{\circ} \mathrm{C}$ (Figures 1(g)-1(i)) appear when we increased the annealing and growth temperatures. Interestingly, hybrid structure composed of one- and two-dimensional carbon allotropes was observed when we employed a moderate cooling rate of $15^{\circ} \mathrm{C} / \mathrm{s}$ regardless of growth temperature (Figure $1(\mathrm{e})$ and insets on Figures $1(\mathrm{~b})$ and $1(\mathrm{~h})$ ), while graphene layers were discovered neither at slower nor at faster cooling rate than $15^{\circ} \mathrm{C} / \mathrm{s}$. From most of the previous studies on the Ni-catalyzed graphene synthesis via precipitation mechanism, it is clearly revealed that a proper cooling process control is critical since the solubility of carbon in $\mathrm{Ni}$ is temperature dependent, and carbon atoms thereby precipitate to form a graphene layer on the surface upon cooling rate $[21,22,30,31]$. Yu et al., in particular, have investigated the cooling rate effect on the formation of the graphene on flat $\mathrm{Ni}$ substrate and reported that: (1) cooling rate faster than $20^{\circ} \mathrm{C} / \mathrm{s}$ results in a carbon quench effect, thus losing the mobility before they can diffuse onto $\mathrm{Ni}$ surface, (2) with slower cooling rate than $0.1^{\circ} \mathrm{C} / \mathrm{s}$, there is enough time for the carbon to diffuse into the bulk, leading to lack of carbon segregation at the surface, and (3) under a medium cooling manipulation, a finite amount of carbon can definitely precipitate to form the graphene at the Ni catalyst surface [21]. Furthermore, Chen et al. amazingly found that single- to multi-layer graphene bridges across the gaps among Ni particles, thus forming a continuous graphene pocket out of agglomerated Ni particles [32]. Considering those facts, it is reasonable to speculate that our continuous graphene layer is precipitated upon a medium cooling rate process out of neighboring $\mathrm{Ni}$ nanoparticles, placed on top of CNT array. In addition, further investigation by energy dispersive X-ray spectra (EDX) analysis was carried out to check both the nanoparticles on top of CNTs and remaining species on bottom substrate. Very few $\mathrm{Ni}$ particles were observed above $\mathrm{Al}$ film on bottom substrate while neither 

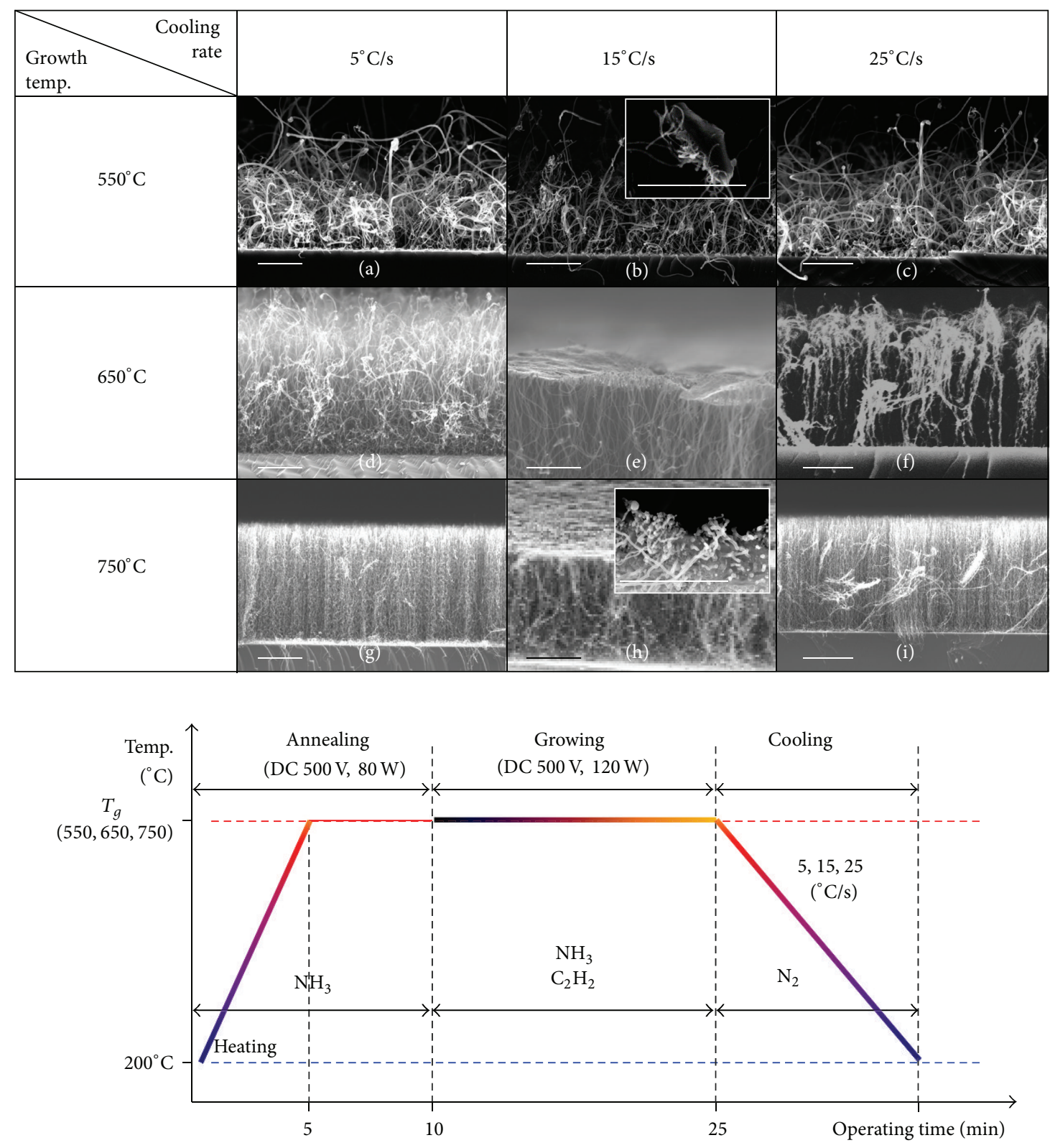

FIGURE 1: Various morphologies of carbon nanostructures with respect to growth temperatures and cooling rates, scale bar: $4 \mu \mathrm{m}$.

Al nor $\mathrm{Al}_{2} \mathrm{O}_{3}$ peak was found on CNT tips, from which we assert MWCNTs are grown with Ni particles by following the tip-growth mechanism and merely $\mathrm{Ni}$ nanoparticles on CNT tips play a role in graphene formation (not shown). To the best of our knowledge, such a fabrication method to form the carbon hybrids has not been reported yet. We thereby paid attention to the characteristics and growth mechanism of such an unusual hybrid carbon nanostructure as seen in Figure 1(e). Figures 2(a)-2(b) indicate our carbon micronymphaea structure comprises a graphene roof on top of vertically aligned MWCNTs, of which the average outer diameter and height measured approximately by $20-25 \mathrm{~nm}$ and $4.7 \mu \mathrm{m}$, respectively (Figures $2(\mathrm{c})-2(\mathrm{~d})$ ). In addition, the precipitated graphene layers have cowrapped all the $\mathrm{Ni}$ domains together with ends of CNTs seen in the magnified image of Figure 2(b), which agreed to Chen et al's report showing a similar morphology of graphene pocket based on the ball-like Ni nanoparticles under the cooling rate of $16.6^{\circ} \mathrm{C} / \mathrm{s}$ [32]. In order to investigate the junction geometry in detail, the hybrid composite was further analyzed by TEM, which provides important information that not only a MWCNT but also graphene is located under the individual $\mathrm{Ni}$ nanoparticle (Figure 2(e) inset), and furthermore graphene layers are bridging across the vicinity to form the continuous layer (left-handed side of dot line) heteroepitaxially as shown in Figure 2(e). This reveals that the graphene could form independently once we grow the dense array of CNTs, thus offering the possibility of the continuous coverage in large scale under an optimized condition. 


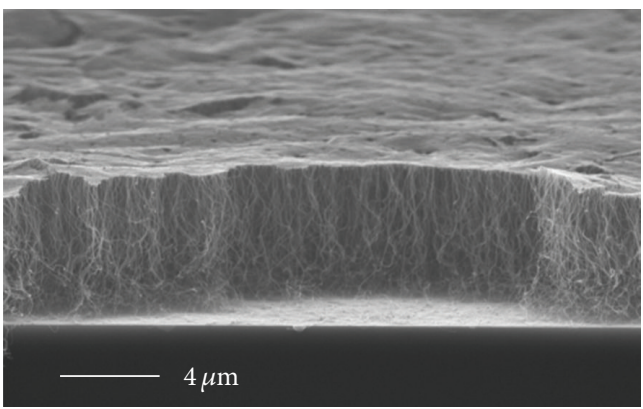

(a)

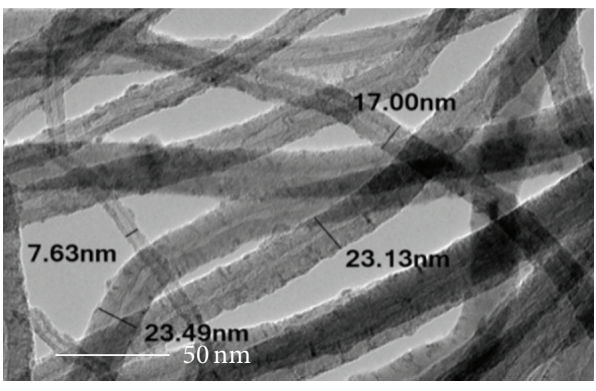

(c)

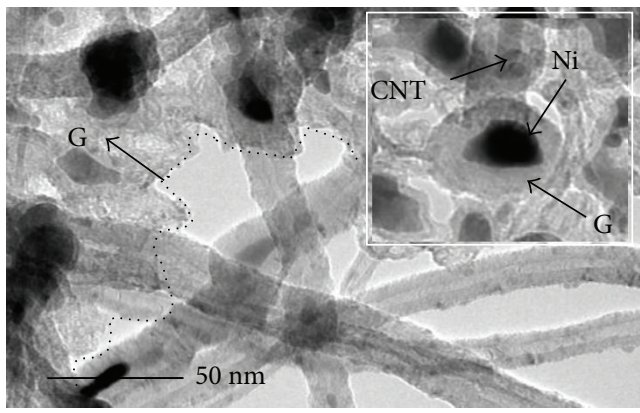

(e)

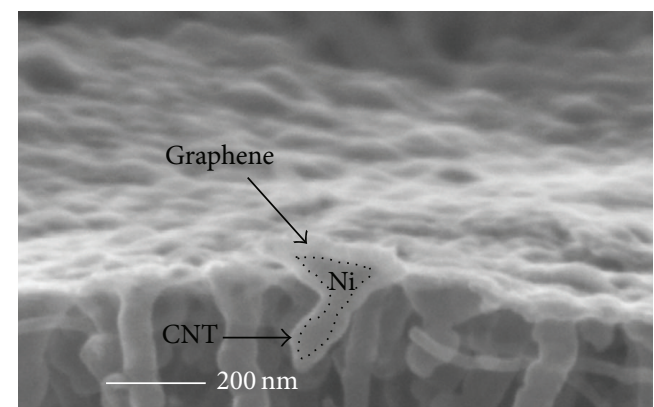

(b)

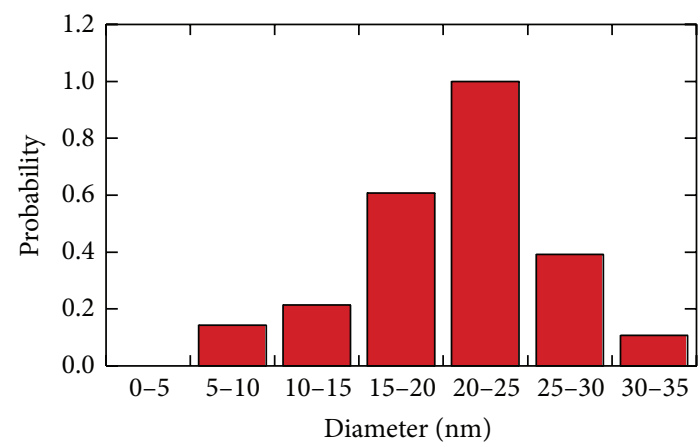

(d)

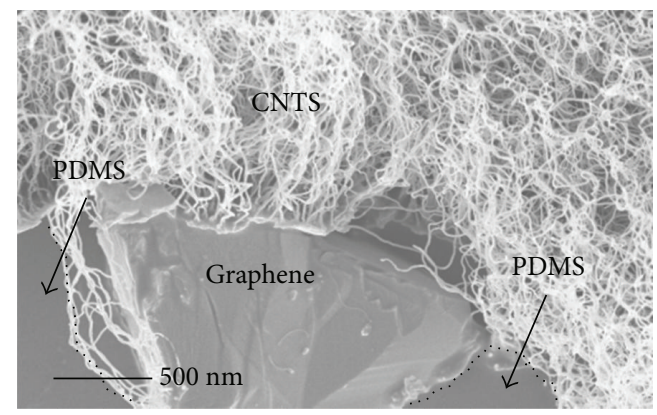

(f)

FIGURE 2: Morphologies of carbon micronymphaea synthesized on $7 \mathrm{~nm} \mathrm{Ni}$ catalyst on $20-\mathrm{nm} \mathrm{Al} \mathrm{film;} \mathrm{(a)} 15^{\circ}$ inclined view, (b) magnified junction between graphene and MWCNTs, (c) TEM image of MWCNT, (d) outer diameter distribution of MWCNTs array, (e) graphene bridging the neighboring $\mathrm{Ni}$ islands, and (f) reversely transferred sample onto PDMS substrate.

On the other hand, for the preparation to check the Raman spectrum of the bare graphene sheet separately out of hybrid composite, we stripped out the hybrid sample from the substrate by stamping with the sticky PDMS film; therefore we could obtain the reverse structure comprised of a graphene layer on PDMS and free standing open MWCNTs upon the graphene as illustrated in Figure 2(f). Cares were taken to focus several spots of exposed bare graphene layer for the investigation of the Raman spectra. Finally, we compared four Raman spectra of the carbon micronymphaea, bare graphene, bare MWCNTs separated from hybrid composite onto $\mathrm{SiO}_{2}$ substrate by a sharp knife, and MWCNTs array grown at the same temperature but faster cooling rate. The black line in Figure 3 indicates the height of $G^{\prime}$ peak $\left(\sim 2681 \mathrm{~cm}^{-1}\right)$ is higher than $\mathrm{G}$ peak $\left(\sim 1596 \mathrm{~cm}^{-1}\right)$ with $\mathrm{G}^{\prime}$ to G Raman peak ratio between 1.43 and 1.53 , corresponding more or less to 4-7 layers according to literatures [28, 29, 33], from which we confirmed the upper layer on CNT array is not a remaining thin Ni layer but the multilayered graphene. In addition, the large D peak is caused from the highly disordered structure when the graphene edges are heteroepitaxially connected [34]. Both green and blue lines show a typical MWCNTs array. The position of G peak $\left(\sim 1590 \mathrm{~cm}^{-1}\right)$ is located at lower wave number than that of bare graphene $\left(\sim 1596 \mathrm{~cm}^{-1}\right)$ since CNT has much higher wall number (mainly 22) compared with the graphene layers (4-7) $[21,35]$. In addition, we report the distinguishable change of G peak $\left(1594.1 \mathrm{~cm}^{-1}\right)$ and $\mathrm{G}^{\prime}$ peak $\left(\sim 2676 \mathrm{~cm}^{-1}\right)$ when MWCNTs array and graphene layers are connected to each other in a carbon micronymphaea structure, which are located at the middle wave number between the bare graphene and CNT (Figure 3, red line). Furthermore, we believe that larger 


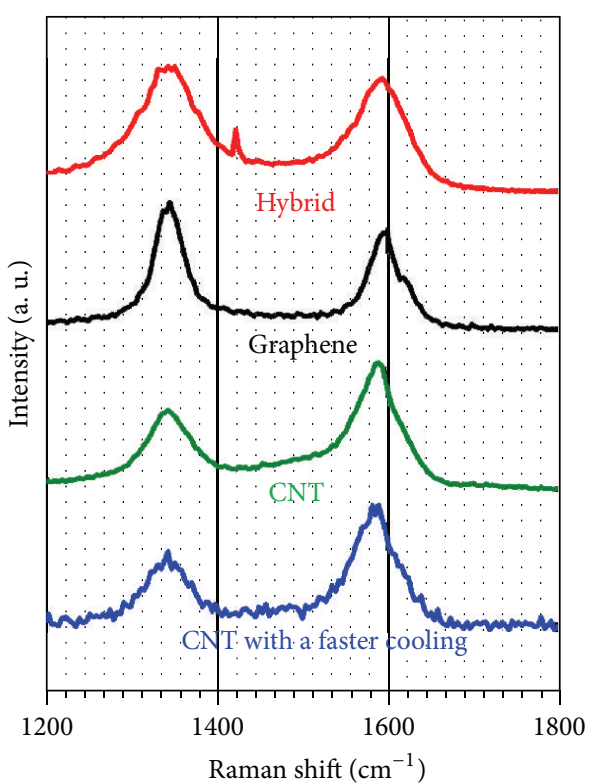

(a)

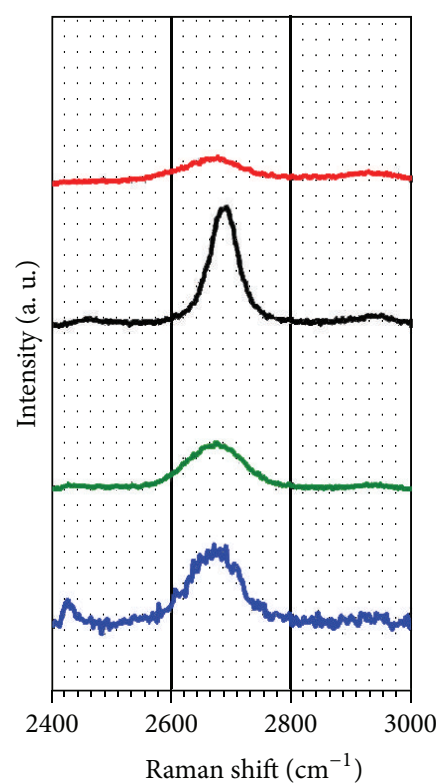

(b)

\begin{tabular}{|c|c|c|c|}
\hline & D peak $\left(\mathrm{cm}^{-1}\right)$ & $\mathrm{G}$ peak $\left(\mathrm{cm}^{-1}\right)$ & $\mathrm{G}^{\prime}$ peak $\left(\mathrm{cm}^{-1}\right)$ \\
\hline Hybrid & 1342.6 & 1594.1 & 2676.6 \\
\hline Graphene & 1343.6 & 1596.1 & 2681.6 \\
\hline CNT from hybrid & 1342.6 & 1590.1 & 2673.1 \\
\hline CNT with & 1342.6 & 1590.1 & 2673.1 \\
\hline a faster cooling & & & \\
\hline
\end{tabular}

(c)

FIGURE 3: Raman spectra at $532 \mathrm{~nm}$ among carbon micronymphaea (red), bare graphene (black) out of hybrids, bare MWCNTs out of hybrids (green), and MWCNTs synthesized at the same growth temperature as hybrids, but under faster cooling rate (blue).

defects of the hybrid are attributed to the discontinuous junction between ends of CNTs and graphene sheet. The junction structure and the Raman spectra need further investigation.

As briefly mentioned in the introduction, there are few previous studies on the synthesis of a carbon hybrid structure with the vertically aligned CNTs based on Co or Ni films using thermal CVD process. These studies proposed that graphene should be first segregated on the surface of continuous thin-film catalyst during increasing the temperature in the presence of a carbon precursor. After the temperature reaches $500-600^{\circ} \mathrm{C}$, the catalyst film under the graphene is broken into particles, and subsequently MWCNTs are synthesized with lifting up the catalyst particles. However, our results suggest a definitely different growth mechanism of the hybrid composite as depicted in Figure 4. First, $7 \mathrm{~nm}$ $\mathrm{Ni}$ film is cloven into nanoparticles during the annealing process in the plasma-induced $\mathrm{NH}_{3}$ surroundings at high temperature. Second, MWCNT array grows via the tipgrowth mechanism, lifting up the $\mathrm{Ni}$ from $\mathrm{Al}$ film at early stage. At this moment, an excessive carbon source is dissolved inside the Ni catalyst. Third, depending upon cooling at an optimal rate, carbon atoms can be precipitated to form graphitic layers along the $\mathrm{Ni}$ islands in lateral directions and subsequently bridge across the gaps between the particles to merge one another heteroepitaxially with the time, which was proved by control experiments in Figure 1. We could not obtain the carbon micronymphaea structure at a cooling rate far from the optimal one. In addition to the cooling rate, we believe that an initial catalyst thickness and annealing time could also affect the graphene quality such as number of layer and the continuity as well as the morphology of vertically aligned CNTs. 

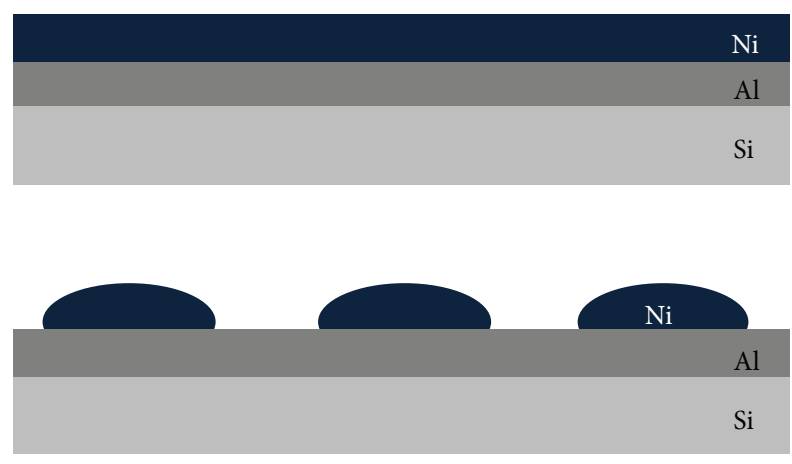

Annealing process

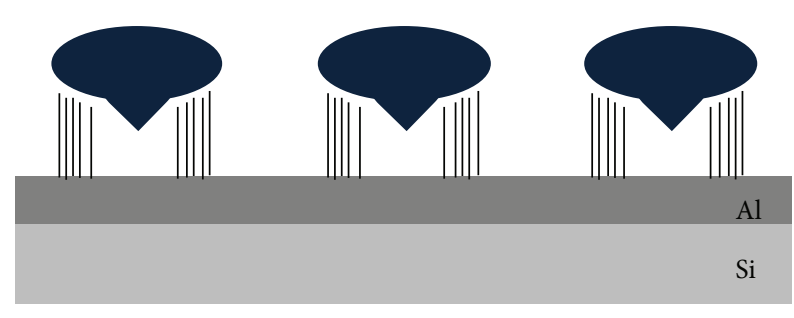

CNTs growth

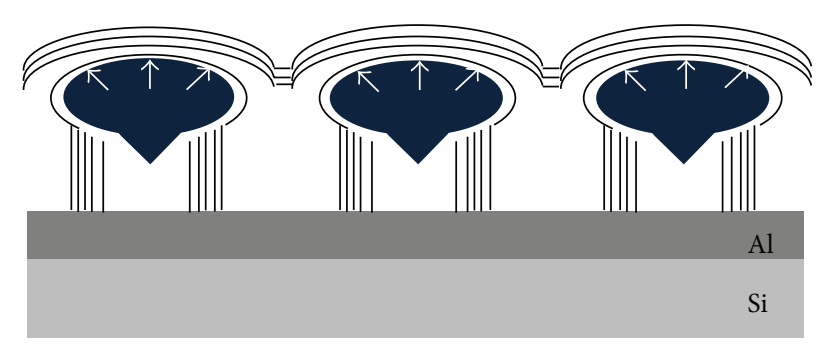

Carbon precipitation to form graphene

FIGURE 4: Proposed growth mechanism of carbon micronymphaea.

\section{Conclusions}

In this study we demonstrated various morphologies of onepot PECVD synthesized carbon nanostructures at different growth temperatures and cooling rates using a set of $7 \mathrm{~nm} \mathrm{Ni}$ layer and $\mathrm{C}_{2} \mathrm{H}_{2}$ precursor. We focused on the characterization and the growth mechanism of a unique carbon hybrid structure, named a carbon micronymphaea; a few layers of graphene are grown on $\mathrm{Ni}$ particles instead of flat substrate and vertically combined with MWCNTs below them. Out of the results, we report when we synthesize CNTs array with the catalyst that is also available to the synthesis of graphene, we can realize either CNTs or CNT/graphene hybrid with respect to the cooling rate, even under the same growth condition. Finally, a carbon micronymphaea is recommended to replace the three-dimensional carbon electrode structure not only to increase the overall specific surface area but also to apply the equipotential to individual CNTs via cowrapped graphene layer.

\section{Acknowledgments}

This work was partially funded by Swiss National Science Foundation (Contracts Nos. 200021-137964 and 200021146856) and Strategic Korean-Swiss Cooperative Program in Science and Technology (FY 2010). The authors gratefully acknowledge the support from the Microfabrication Center (FIRST), the Electron Microscopy Center (EMEZ), and Professor C. Hierold of ETH Zurich. Dr. E. B. Meier's help in transmission electron microscopy is greatly recognized. Hyung Gyu Park and Seul Ki Youn acknowledge their travel support from COST action MP0901 NanoTP.

\section{References}

[1] M. S. Purewal, B. H. Hong, A. Ravi, B. Chandra, J. Hone, and P. Kim, "Scaling of resistance and electron mean free path of single-walled carbon nanotubes," Physical Review Letters, vol. 98, no. 18, Article ID 186808, 2007.

[2] W.-K. Tse, E. H. Hwang, and S. Das Sarma, "Ballistic hot electron transport in graphene," Applied Physics Letters, vol. 93, no. 2, Article ID 023128, 2008.

[3] S. D. Li, Z. Yu, C. Rutherglen, and P. J. Burke, "Electrical properties of $0.4 \mathrm{~cm}$ long single-walled carbon nanotubes," Nano Letters, vol. 4, no. 10, pp. 2003-2007, 2004.

[4] F. Giannazzo, S. Sonde, R. L. Nigro, E. Rimini, and V. Raineri, "Mapping the density of scattering centers limiting the electron mean free path in graphene," Nano Letters, vol. 11, no. 11, pp. 4612-4618, 2011.

[5] R. F. Zhang, Q. Wen, W. Z. Qian, D. S. Su, Q. Zhang, and F. Wei, "Superstrong ultralong carbon nanotubes for mechanical energy storage," Advanced Materials, vol. 23, no. 30, pp. 33873391, 2011.

[6] C. Lee, X. D. Wei, J. W. Kysar, and J. Hone, "Measurement of the elastic properties and intrinsic strength of monolayer graphene," Science, vol. 321, no. 5887, pp. 385-388, 2008.

[7] E. Pop, D. Mann, Q. Wang, K. Goodson, and H. Dai, “Thermal conductance of an individual single-wall carbon nanotube above room temperature," Nano Letters, vol. 6, no. 1, pp. 96-100, 2006.

[8] A. A. Balandin, S. Ghosh, W. Bao et al., "Superior thermal conductivity of single-layer graphene," Nano Letters, vol. 8, no. 3, pp. 902-907, 2008.

[9] S. Paulson, A. Helser, M. Buongiorno Nardelli et al., "Tunable resistance of a carbon nanotube-graphite interface," Science, vol. 290, no. 5497, pp. 1742-1744, 2000.

[10] H. Q. Zhu, Y. M. Zhang, L. Yue et al., "Graphite-carbon nanotube composite electrodes for all vanadium redox flow battery," Journal of Power Sources, vol. 184, no. 2, pp. 637-640, 2008.

[11] Y. Tang and J. H. Gou, "Synergistic effect on electrical conductivity of few-layer graphene/multi-walled carbon nanotube paper," Materials Letters, vol. 64, no. 22, pp. 2513-2516, 2010.

[12] U. Khan, I. O'Connor, Y. K. Gun'Ko, and J. N. Coleman, “The preparation of hybrid films of carbon nanotubes and nanographite/graphene with excellent mechanical and electrical properties," Carbon, vol. 48, no. 10, pp. 2825-2830, 2010.

[13] D. S. Yu and L. M. Dai, "Self-assembled graphene/carbon nanotube hybrid films for supercapacitors," Journal of Physical Chemistry Letters, vol. 1, no. 2, pp. 467-470, 2010. 
[14] B. A. Zhang, Q. B. Zheng, Z. D. Huang, S. W. Oh, and J. K. Kim, " $\mathrm{SnO}_{2}$-graphene-carbon nanotube mixture for anode material with improved rate capacities," Carbon, vol. 49, no. 13, pp. 45244534, 2011.

[15] C. Y. Li, Z. Li, H. W. Zhu et al., "Graphene nano-"patches" on a carbon nanotube network for highly transparent/conductive thin film applications," Journal of Physical Chemistry C, vol. 114, no. 33, pp. 14008-14012, 2010.

[16] D. Kondo, S. Sato, and Y. Awano, "Self-organization of novel carbon composite structure: graphene multi-layers combined perpendicularly with aligned carbon nanotubes," Applied Physics Express, vol. 1, no. 7, Article ID 0740033, 2008.

[17] V. Jousseaume, J. Cuzzocrea, N. Bernier, and V. T. Renard, "Few graphene layers/carbon nanotube composites grown at complementary-metal-oxide-semiconductor compatible temperature," Applied Physics Letters, vol. 98, no. 12, Article ID 123103, 2011.

[18] R. Martel, T. Schmidt, H. R. Shea, T. Hertel, and P. Avouris, "Single- and multi-wall carbon nanotube field-effect transistors," Applied Physics Letters, vol. 73, no. 17, pp. 2447-2449, 1998.

[19] C. H. Yu, L. Shi, Z. Yao, D. Li, and A. Majumdar, "Thermal conductance and thermopower of an individual single-wall carbon nanotube," Nano Letters, vol. 5, no. 9, pp. 1842-1846, 2005.

[20] D. Takagi, Y. Homma, H. Hibino, S. Suzuki, and Y. Kobayashi, "Single-walled carbon nanotube growth from highly activated metal nanoparticles," Nano Letters, vol. 6, no. 12, pp. 2642-2645, 2006.

[21] Q. K. Yu, J. Lian, S. Siriponglert, H. Li, Y. P. Chen, and S.-S. Pei, "Graphene segregated on Ni surfaces and transferred to insulators," Applied Physics Letters, vol. 93, no. 11, Article ID 113103,2008

[22] K. S. Kim, Y. Zhao, H. Jang et al., "Large-scale pattern growth of graphene films for stretchable transparent electrodes," Nature, vol. 457, no. 7230, pp. 706-710, 2009.

[23] A. Reina, X. T. Jia, H. John et al., "Layer area, few-layer graphene films on arbitrary substrates by chemical vapor deposition," Nano Letters, vol. 9, pp. 3087-3087, 2009.

[24] M. Cantoro, S. Hofmann, S. Pisana et al., "Effects of pretreatment and plasma enhancement on chemical vapor deposition of carbon nanotubes from ultra-thin catalyst films," Diamond and Related Materials, vol. 15, no. 4-8, pp. 1029-1035, 2006.

[25] R. R. Mitchell, B. M. Gallant, C. V. Thompson, and Y. Shao-Horn, "All-carbon-nanofiber electrodes for high-energy rechargeable Li- $\mathrm{O}_{2}$ batteries," Energy and Environmental Science, vol. 4, no. 8, pp. 2952-2958, 2011.

[26] M. Bikshapathi, A. Sharma, A. Sharma, and N. Verma, "Preparation of carbon molecular sieves from carbon micro and nanofibers for sequestration of $\mathrm{CO}_{2}$," Chemical Engineering Research and Design, vol. 89, no. 9, pp. 1737-1746, 2011.

[27] C. T. Wirth, S. Hofmann, and J. Robertson, "State of the catalyst during carbon nanotube growth," Diamond and Related Materials, vol. 18, no. 5-8, pp. 940-945, 2009.

[28] D. Graf, F. Molitor, K. Ensslin et al., "Spatially resolved raman spectroscopy of single- and few-layer graphene," Nano Letters, vol. 7, no. 2, pp. 238-242, 2007.

[29] A. Das, B. Chakraborty, and A. K. Sood, "Raman spectroscopy of graphene on different substrates and influence of defects," Bulletin of Materials Science, vol. 31, no. 3, pp. 579-584, 2008.
[30] A. N. Obraztsov, E. A. Obraztsova, A. V. Tyurnina, and A. A. Zolotukhin, "Chemical vapor deposition of thin graphite films of nanometer thickness," Carbon, vol. 45, no. 10, pp. 2017-2021, 2007.

[31] H. J. Park, J. Meyer, S. Roth, and V. Skákalová, “Growth and properties of few-layer graphene prepared by chemical vapor deposition," Carbon, vol. 48, no. 4, pp. 1088-1094, 2010.

[32] Z. P. Chen, W. C. Ren, B. L. Liu et al., "Bulk growth of monoto few-layer graphene on nickel particles by chemical vapor deposition from methane," Carbon, vol. 48, no. 12, pp. 35433550, 2010.

[33] A. C. Ferrari, J. C. Meyer, V. Scardaci et al., "Raman spectrum of graphene and graphene layers," Physical Review Letters, vol. 97, no. 18, Article ID 187401, 2006.

[34] A. C. Ferrari, "Raman spectroscopy of graphene and graphite: disorder, electron-phonon coupling, doping and nonadiabatic effects," Solid State Communications, vol. 143, no. 1-2, pp. 47-57, 2007.

[35] J. M. Caridad, F. Rossella, V. Bellani, M. Maicas, M. Patrini, and E. Díez, "Effects of particle contamination and substrate interaction on the Raman response of unintentionally doped graphene," Journal of Applied Physics, vol. 108, no. 8, Article ID 084321, 2010 

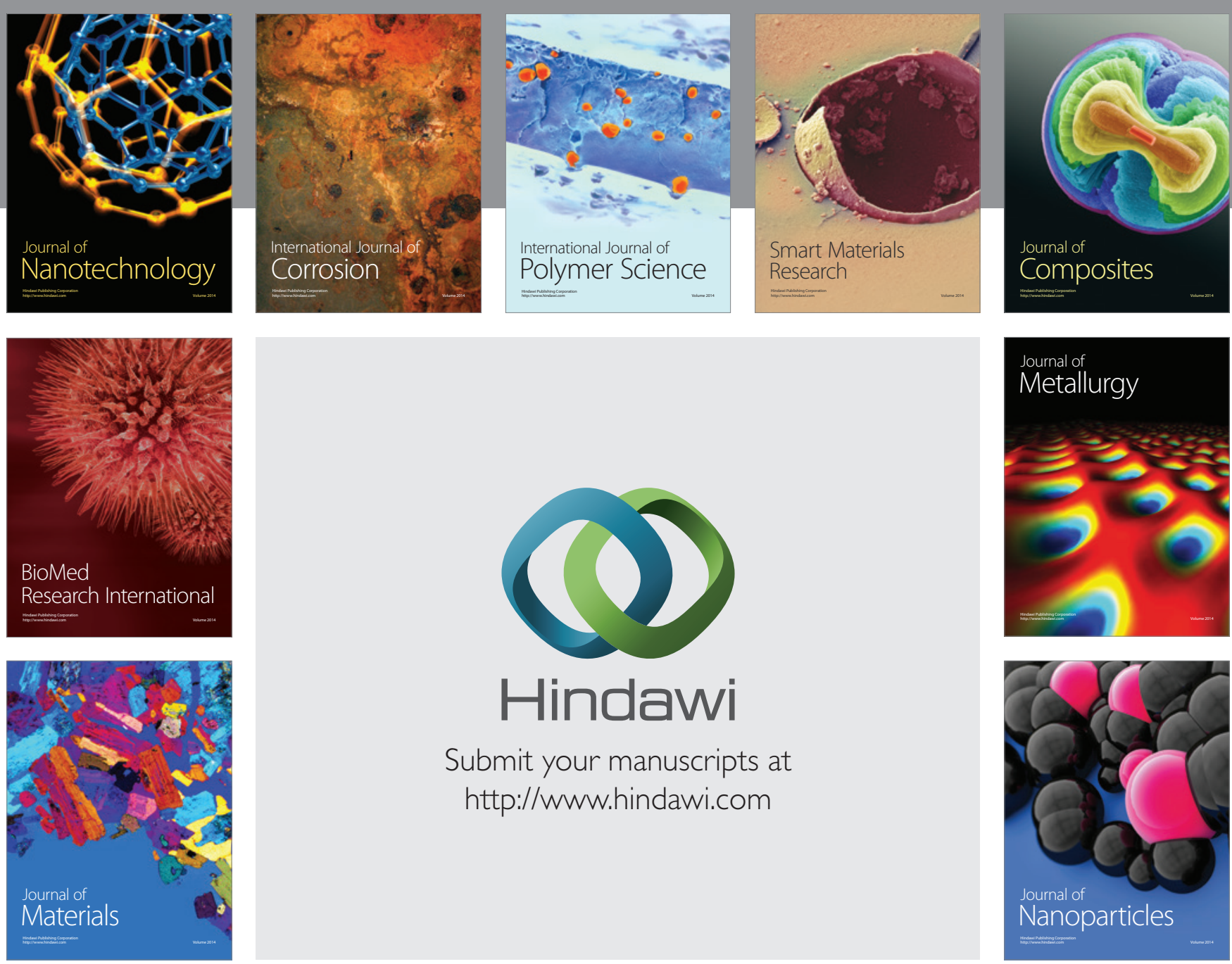

Submit your manuscripts at http://www.hindawi.com
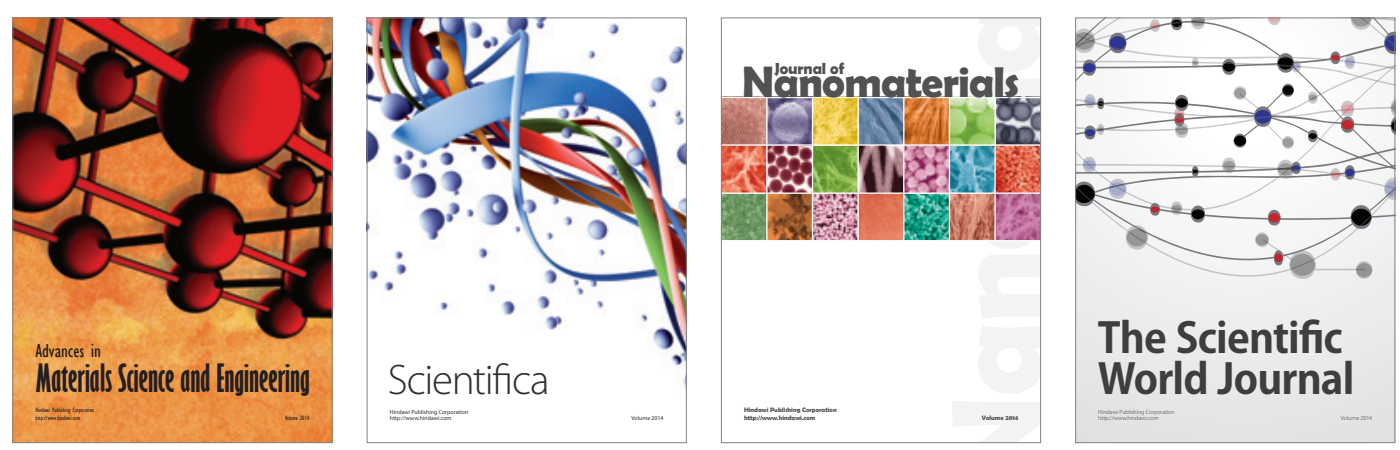

\section{The Scientific World Journal}
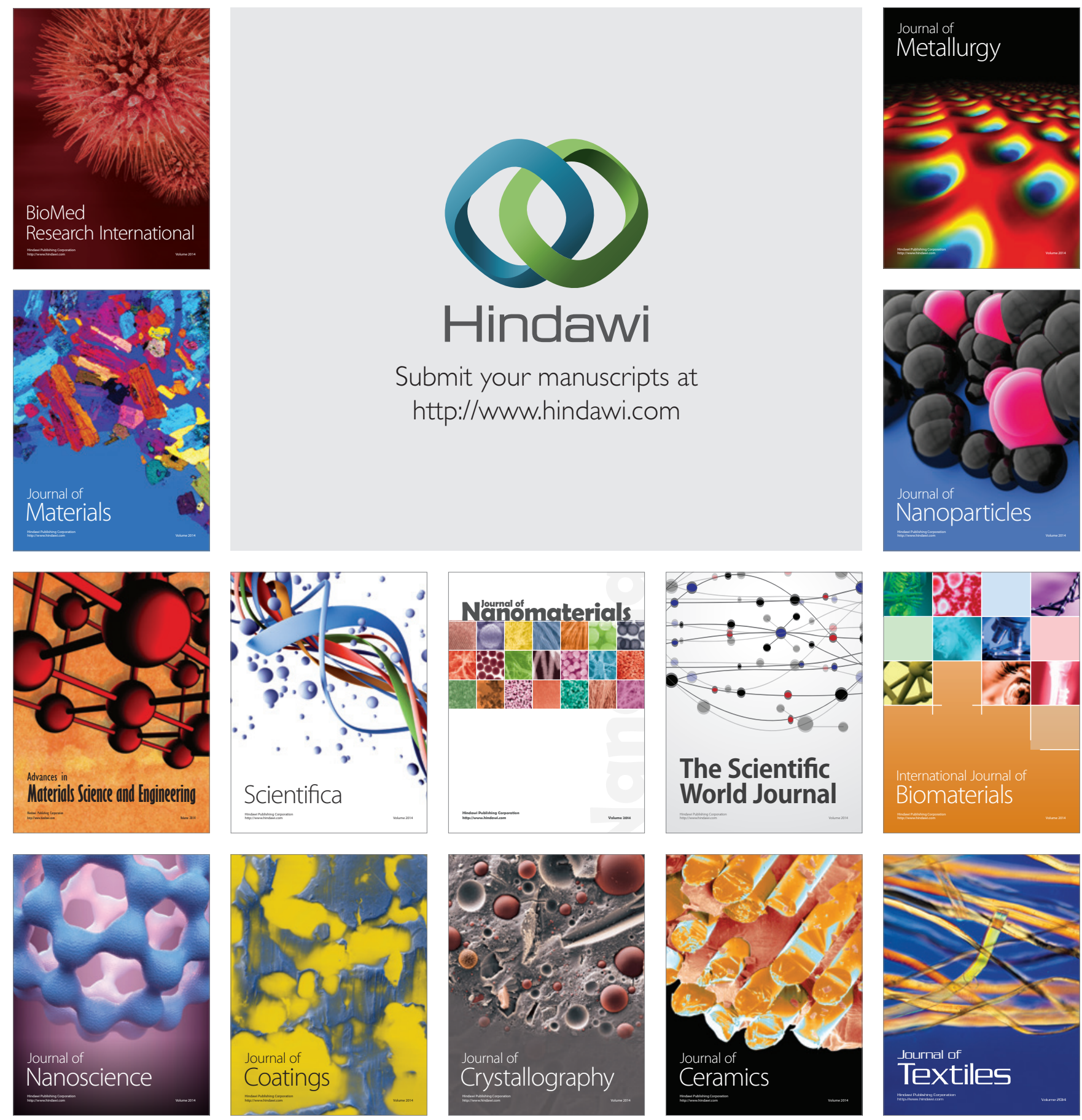